\title{
Psychiatric Comorbidity Related to the Therapy of Male Androgenetic Alopecia Independent of the 5-Alpha Reductase Pathway
}

\author{
Ralph M. Trüeb \\ Center for Dermatology and Hair Diseases, Zurich-Wallisellen, Switzerland
}

Dear Editor,

I have recently commented on psychological adverse events in male patients treated with oral finasteride, specifically the post-finasteride syndrome (PFS) [1]. Individuals suffering from PFS present with symptoms of persistent sexual, mental, and physical side effects despite stopping finasteride.

Neuroendocrinological research has provided the scientific evidence for the PFS from studies in rodents, in which finasteride may reduce the concentration of neuroactive steroids. Indeed, an important neurosteroid is allopregnanolone (ALLO), a metabolite of dihydroprogesterone, and potent ligand of the inhibitory GABA-barbiturate receptor. GABA receptors have variable sensitivities to ALLO in the settings of neurosteroid withdrawal, stress, and social isolation. Less ALLO, as a consequence of finasteride treatment, could alter GABAergic transmission with effects on neurogenesis and neuronal survival. Notably, the mechanisms of irreversible tardive dyskinesias from phenothiazines may be similar to those underlying the PFS: in rats treated with haloperidol to induce orofacial dyskinesia, co-administration of progesterone prevented this side effect, while pre-treatment with finasteride reversed this protective effect, demon- strating an important role of the progesterone pathway [2]. Since neurosteroids are believed to have anxiolytic and antidepressant properties, the decrease of neurosteroid biosynthesis through inhibition of 5-alpha-reductase required for neurosteroid synthesis, may contribute to the respective psychiatric adverse events. And yet, a group of hair experts who are among the most frequent prescribers of finasteride for treatment of androgenetic alopecia (AGA) have scrutinized the literature on the PFS on the backdrop of their experience, and not found the condition to be plausible as it has emerged from reports of non-dermatologists, neuroendocrinological research, and so far has only been documented in low-quality studies with a strong bias selection [3].

Ultimately, I reported a case of PFS after 20 years of successful prescription practice of finasteride for treatment of AGA, with circumstantial evidence that the PFS may rather represent a delusional disorder of the somatic type than an endocrinological disorder, possibly on the background of a histrionic personality disorder. In fact, the PFS demonstrates analogies to other mystery syndromes, such as amalgam illness, multiple chemical sensitivity, Morgellons disease, and Koro, in which the alleged symptoms cannot be explained biologically, and the 
frequency of consultations for the respective condition parallels the media coverage, which points to a high degree of suggestibility [4].

Finally, I was recently consulted by a 29 -year-old male who complained of depression and restlessness within 2 months of b.i.d. application of a commercial 5\% topical minoxidil solution (Regaine ${ }^{\circledR}$ ) prescribed by the general practitioner. The symptoms have persisted despite cessation of the therapy. The patient pointed out that to his knowledge, the product insert lists depression among the undesired effects.

Typically, patients with the PFS complain of persistent symptoms of depression and associated sexual disorders, despite cessation of therapy with finasteride and independent of the duration of finasteride therapy. There is a high degree of suggestibility and nocebo effect.

This case observation is the first to demonstrate yet another example of persistent psychiatric comorbidity related to the therapy of male AGA, however independent of the 5-alpha-reductase pathway, suggesting that men suffering from AGA may be at a particular risk. Preexisting mental health disorder may put patients at an increased risk, while the prevalence of personality disorders in subjects with AGA has been shown to be significantly higher than in the general population [5]. Ultimately, the psychological effects of alopecia may be hard to differentiate from preexisting psychopathology. Patients with personality disorders experience more distress from alopecia than non-disordered patients, since these individuals lack a secure sense of self and effective coping skills, and therefore may be especially vulnerable to the adverse effects of both alopecia and treatment, depending on the extent and type of coverage in the social and print media.

\section{Statement of Ethics}

There are no ethical conflicts to disclose.

\section{Conflict of Interest Statement}

None declared.

\section{Funding Sources}

None declared.

\section{References}

1 Trüeb RM, Reis G, Dias MF, Dutra Rezende H. Comment on suicidality and psychological adverse events in patients treated with finasteride. Skin Appendage Disord. 2021. In press.

2 Irwig MS. Persistent sexual side effects of finasteride: could they be permanent? J Sex Med. 2012;9(11):2927-32.
3 Fertig R, Shapiro J, Bergfeld W, Tosti A. Investigation of the plausibility of 5-alpha-reductase inhibitor syndrome. Skin Appendage Disord. 2017;2(3-4):120-9.

4 Trüeb RM, Régnier A, Dutra Rezende H, Gavazzoni Dias MFR. Post-finasteride syndrome: an induced delusional disorder with the potential of a mass psychogenic illness? Skin Appendage Disord. 2019 Aug;5(5):320-6.
5 Maffei C, Fossati A, Rinaldi F, Riva E. Personality disorders and psychopathologic symptoms in patients with androgenetic alopecia. Arch Dermatol. 1994;130(7):868-72. 